\title{
Ecological importance and invertebrate conservation
}

\author{
T. R. New and A. L. Yen
}

The immense ecological importance of invertebrate animals is discussed in relation to using ecological values in setting priorities for their conservation. The spectrum of values, from taxa that we know perform vital ecological roles to taxa that may be ecologically redundant, is outlined.

\section{Introduction}

Invertebrates are the most diverse animals in the world's natural communities, be they marine, freshwater or terrestrial, and are acknowledged widely as playing central roles in sustaining those systems. Wilson's (1987) encapsulation of invertebrates as 'the little things that run the world' emphasizes their importance. Invertebrate well-being and ecological sustainability are probably linked intricately in many different ways, and an appreciation of this is vital in promoting the need to conserve invertebrates and in helping to guide practical priorities for achieving it. Ecological importance, sometimes emphasized by description of particular taxa as 'keystone species', is a criterion used to focus attention on particular taxa and select particular taxonomic groups as tools in broader conservation management. Defining the ecological importance of invertebrates, however, is often difficult in other than simplistic terms or theoretical ideals, not least because the diversity of highly localized and ecologically specialized invertebrates in any given community tends to be high. This makes clarifying their relative roles there an exercise peculiar to that community; it can be guided only in part from experiences elsewhere. Loss of relatively specific invertebrate complements might be catastrophic for the sustainability of the community in which they participate.

A major current strategy for invertebrate conservation follows the major emphasis for vertebrate conservation in concentrating on particular species. One possible consequence of continuing to focus on individual species for conservation among invertebrates in the current, somewhat ad hoc, manner is that they might not be those that are most important ecologically. By concentrating a high proportion of our logistic capability on individual species, the vast unseen majority of species might still be disappearing, and the systems that they constitute might eventually collapse. This criticism differs from the more common one, that focusing on species of invertebrates for conservation is 'merely tinkering around the edges', but the outcomes may be similar. Our resources will never be sufficient to give individual attention to all invertebrate species that need conservation action. The major challenge in conserving 'biodiversity' (dominated by invertebrate animals) is, perhaps, not simply to save species $X$ or species $Y$, but to curtail or slow the massive rates of extinction that are anticipated to occur over the next few decades. At the same time, individual species will remain of immense value as 'flagships' for conservation, and as tangible entities to which people can relate easily. For example, in many countries individual butterfly species have been used to publicize the needs of insect conservation and to make people aware, for the first time, that invertebrates need management in order to sustain them. However, the ecological importance of many such flagship species is most usually unknown, and may sometimes be small.

This paper discusses how 'ecological importance' might be investigated, and 
whether it might help in improving the focus for invertebrate conservation.

\section{Ecological importance}

People usually appreciate the ecological importance of particular invertebrate taxa in contexts to which they can relate easily; honeybees as important pollinators of orchard and agricultural crops, earthworms as decomposers and soil-conditioners, and various arthropods as biological control agents for pests have widely recognized positive values based on their ecological roles (Council of Europe, 1986; Kellert, 1993) and these examples are valuable in promoting conservation of invertebrates. In most natural communities, invertebrates are not as obviously associated with human well-being, but their roles are just as vital. In the context of dry forests of Costa Rica, Janzen (1987) discussed the largely undocumented ways in which insects are part of the 'glue and building blocks' of natural ecosystems. These are listed below.

1 Many predators, such as insectivorous vertebrates, select particular kinds of prey to which they are in some way adapted. In Janzen's words 'insect species are not merely interchangeable bits of nutrients'.

2 Two families of beetles (Curculionidae, Bruchidae) destroy a very high proportion of legume seeds. If the beetles disappeared, it is possible that particular legume species could become superabundant because a major impediment to their reproduction would have been removed. One particular weevil species consumed more than 80 per cent of seeds of a tree species characteristic of secondary succession. If this loss did not occur such plants might be pervasive invaders of the forest (Janzen, 1987).

3 Many pollinator-plant relationships are highly specialized but even highly specific insect pollinators might not be permanent residents in the community. Many hawkmoths (Sphingidae) in Costa Rica are migrants and spend part of the year in other habitats, which might become threatened.

Similar scenarios could be expounded from elsewhere and for a wide variety of invertebrate phyla. Importance of an invertebrate group might be evident through its taxonomic diversity. In Australia, for example, the moth family Oecophoridae is one of the largest insect groups, with nearly 6000 species (Common, 1990). Most species are associated with Eucalyptus and related genera of trees, and caterpillars of many oecophorids feed on dead fallen foliage. They are among the predominant decomposer taxa for otherwise persistent eucalypt litter and appear to have radiated to exploit this resource very effectively. In other assemblages single species might be vital. In Antarctic oceans, krill (Euphausia superba, and a few related species) is a major link in the relatively simple predominant food webs. The importance of krill might appear relatively obvious because of its great abundance and biomass and lack of other taxa occupying a similar role, but many 'unseen' invertebrates such as the Oecophoridae clearly play roles that appear to be necessary to maintain dynamic balance in natural communities.

It may, therefore, be important to distinguish such 'vital taxa', if they can indeed be distinguished; if they can, there is clear implication that 'some species are more important than others'. The predominance of invertebrates has raised two themes that are becoming increasingly important in attempting to define ecological roles in relation to setting conservation priorities. First, can/do invertebrates act as keystone taxa? Second, are any of them ecologically redundant? Both concepts are controversial.

\section{Keystone taxa}

The existence of 'keystone taxa', species or higher groupings on which many others in a community depend and without which the community could not persist, is difficult to confirm. Despite the appealing nature of the concept, some species named as keystones are simply those that have been studied reasonably fully. Many such taxa have been suggested in only general terms, and critical 
experiments involving the removal of putative keystone taxa and monitoring the fate of the impoverished community are extraordinarily difficult (perhaps, even, irresponsible) to undertake. Nevertheless, any such taxa or guilds by definition may merit priority for conservation. One consequence of their loss is likely to be 'cascading secondary extinctions' (Allan and Flecker, 1993), in themselves also difficult to document.

Krill is a putative keystone species group in Antarctic marine environments. Termites (Isoptera) might comprise a keystone invertebrate group in arid inland Australia; Morton and James (1988) suggested that the high diversity of lizard species there might be due to the abundance of termites. Lizard diversity is especially high in infertile spinifex grasslands and acacia-dominated shrublands, and declines with increasing rainfall and soil fertility elsewhere. The general low soil fertility and sporadic production of plant material in the arid areas appears to favour termites over other grazing and detritivorous animals. Termites are a major food item for the lizards, which may have specialized behaviour patterns (such as being active at night) that increase their harvesting efficiency. Other invertebrate groups preying on termites could also become more abundant and constitute additional food for the lizards. Although the scenario is complex (Pianka, 1989) there is strong suggestion of a central role for termites in this system.

Krill and termites are 'high abundance' invertebrate taxa. However, central (keystone) roles need not necessarily equate with being common. A specific pollinator of a vital plant, for example, could be quite rare.

\section{Ecological redundancy}

In contrast with the central roles played by keystone taxa, the implication of ecological redundancy is that some species may not be of any significant ecological importance and that their loss will not jeopardize the functioning of the communities that contained them. In many cases, such taxa might already be rare (and, as such, might in other contexts be ranked highly for conservation attention). If their trophic roles overlap with other taxa in the same assemblage, any 'slack' created by their loss may be buffered adequately by other taxa, and the community remain sustainable.

\section{Discussion}

Either of these extremes may not apply to most of the invertebrate species present, and the widespread view errs on the side of caution: that loss of any species may be harmful, and that if this process proceeds there will be - at some unspecified level of extinctions - insufficient ecological capability left to maintain the community or ecosystem in its pristine functional state. Defining which species may be important, or vital, in an assemblage is extraordinarily difficult, as is assessing the amount of species loss that could be tolerated.

The two ideas are combined in Walker's (1992) analogy that some species may be viewed as the 'drivers' of an ecosystem, whereas some others are merely 'passengers'. Keystone (or driver) taxa must be conserved for sustainability whereas - in functional, if not ethical, terms - 'passengers' might be deemed expendable and given lower priority for conservation, simply because their loss would not destroy the community's integrity. In the history of conservation practice, recognition that any invertebrates are more than passengers when considered in relation to mammals, birds or large plants, is itself still novel to many managers. In some instances of modified or simplified systems, 'drivers' (or presumed drivers) may need to be introduced or managed actively. Much of the historical wealth of Australia has come through agricultural development, for example, and one consequence of the massive conversion of lands from natural vegetation to cropping or pastoral use has been range contraction of many native earthworms. This has been countered in part by introduction of exotic earthworm species, which are now far more important than native species in sustaining productivity of some agricultural systems (Baker, 1992). 
This situation is not equivalent to conserving natural communities, of course, but indicates the importance of maintaining key invertebrate functions and roles when any systems are modified for human use.

Detecting and delimiting the relative roles of invertebrates in communities is far from easy. When trying to detect ecological redundancy, Walker (1992) suggested the approach of guild analysis, categorizing the taxa present into their major ecological roles to indicate the number of species in each. A guild with many species might be able to lose taxa without impairing its overall function. Guilds with few species may not be able to withstand any loss if their collective role is to be maintained and such species should perhaps be given conservation priority over members of more speciose guilds. Any available evidence on the interactions between species within a guild (including their capability for density compensation if taxa are lost) may indicate ecological stability. This approach is extraordinarily difficult to put into practice, not least because we know so little about the feeding habits of invertebrates, and the extent to which specializations within any guild are needed to maintain the guild's overall function in an assemblage. Several published studies of presumptive guild analysis of insect assemblages, for example, have extrapolated knowledge of a few species to whole families or orders, assuming ecological homogeneity (which might not exist), or have allocated trophically complex taxa to a single ecological role. The degree of feeding specificity of members of any guild is usually unknown - for example, which of the herbivorous insects are monophagous and which feed on many kinds of plants and, therefore, the extent to which members of a guild may compete or otherwise interact with each other. Likewise, the relationships between the species present and the stability of the ecosystem are poorly understood. In short, we do not know which, if any, species are expendable and conservation of as many species as possible would appear to be by far the wisest strategy to pursue.

Models by Pimm (1986) imply that removal of predators from species-rich systems has wide-ranging destabilizing effects on the rest of the community. However, from this it is a large leap to suggesting that all such predators are necessarily essential, or are keystone taxa. Degraded ecosystems may be apparent mainly by changes in the species assemblage composition rather than simply by harbouring few species. A loss of specialist species and their replacement by relative generalists may maintain an overall capability for sustaining processes. However, it may involve the loss of many of the specialized nuances needed to prevent further loss of taxa and to buffer the ecosystem against further changes. Secondary forest areas in Papua New Guinea, for example, support a very high proportion of the butterfly species recorded in the region (Parsons, 1992), but loss of primary forest there will still lead to loss of notable specialist species that cannot withstand changed conditions. For groups that are less conspicuous or less well-documented than butterflies, equivalent knowledge is usually not available.

We do not pretend to have solutions to the problems of quantifying the ecological worth of particular invertebrates. But, as Kellert (1993) and others have emphasized, the stability and integrity of most ecosystems, and their resilience to anthropogenic change, is due in no small measure to invertebrate animals, and various adverse ecological effects have resulted from their loss. We do not know enough to claim that any species is 'ecologically redundant', and the challenge remains to incorporate ecological values meaningfully into practical conservation of invertebrate assemblages. At the least, widespread realization that many invertebrates are fundamentally important in maintaining the systems on which humans depend may help to reduce the levels of apathy and hostility with which invertebrates are currently regarded. Extending Walker's (1992) analogy, first-class passengers might contribute more to the community's economy than third-class passengers do but, if they all sit together, we may not be able to tell which is which without improving our ticket inspection capability. And if the first-class passengers, or enough of the third-class 
passengers to make an equivalent contribution, disembark, the system may well falter or fail even if the driver remains fit. The quality of the passengers may indeed be important in invertebrate assemblages, and as long as we are unable to discriminate between importance of taxa, protection of habitats to ensure as little loss of taxa as possible is the paramount need for conserving invertebrates.

\section{References}

Allan, J.D. and Flecker, A.S. 1993. Biodiversity conservation in running waters. BioScience, 43, 32-43.

Baker, G.H. (ed.) 1992. Earthworm Research in Australia, 1991-92. CSIRO, Adelaide.

Common, I.F.B. 1990. Moths of Australia. Melbourne University Press, Melbourne.

Council of Europe 1986. European Charter for Invertebrates. Strasbourg.

Janzen, D.H. 1987. Insect diversity of a Costa Rican dry forest: why keep it, and how? Biol. J. Linn. Soc., 30, 343-356.
Kellert, S.R. 1993. Values and perceptions of invertebrates. Conservation Biology, 7, 845-855.

Morton, S.R. and James, C.D. 1988. The diversity and abundance of lizards in arid Australia: a new hypothesis. Amer. Nat., 132, 237-257.

Parsons, M.J. 1992. Butterflies of the Bulolo-Wau Valley. Bishop Museum Press, Honolulu.

Pianka, E.R. 1989. Desert lizard diversity: additional comments and some data. Amer. Nat., 134, 344-364.

Pimm, S.L. 1986. Community stability and structure. In Conservation Biology (ed. M. E. Soulé), pp. 309-329. Sinauer, Sunderland, Mass.

Walker, B.H. 1992. Biodiversity and ecological redundancy. Conservation Biology, 6, 18-23.

Wilson, E.O. 1987. The little things that run the world (the importance and conservation of invertebrates). Conservation Biology, 1, 344-347.

T. R. New, School of Zoology, La Trobe University, Bundoora, Victoria 3083, Australia.

A. L. Yen, Museum of Victoria, 71 Victoria Crescent, Abbotsford, Victoria 3087, Australia. 